\title{
An Organizational Theory of Information
}

\author{
Anatol W. Holt and Felice Cardone \\ DISCo, Università di Milano-Bicocca, Via Bicocca degli Arcimboldi 8, I-20126 Milano, Italy \\ e-mail: anatolholt@popmail.iol.it,cardone@disco.unimib.it
}

Key words: Information, Organization, Plan.

Abstract: This paper briefly presents a new technical and philosophical approach to "information" as relevant to computerized society. Our definition of "information" makes no reference to computers, but makes extensive reference to organized activities within a society affected. Specific problems that particularly impact computer use are indicated. The subject concept "information" is compared to others that have had influence in the past. The paper is less a contribution to an established direction in practice and research than it is direction setting.

\section{INTRODUCTION}

For half a century it has been clear that computers are, above all, "information machines"; as users we let information flow in and out, store it, transform it, search, discard and view it. Nevertheless - although information is so important to computers, and computers are so important to our society - no new concepts of "information" have guided users or designers into the future. A theory of information taking into account the variety of information-relative operations that can be performed with the help of computers is likely to have to the computer the same relation as thermodynamics to the steam engine. This can hardly be the case for classical information theory, as developed originally by Shannon (Shannon

The original version of this chapter was revised: The copyright line was incorrect. This has been corrected. The Erratum to this chapter is available at DOI: 10.1007/978-0-387-35500-9_30 
and Weaver, 1949), motivated by the needs of telephony and focussed essentially on the (efficiency of) transmission of information.

In our culture people assume (consciously or unconsciously) that information is objective - simply there, like the center of mass of the solar system (to borrow an example from Frege). This attitude is supported by current theories of information, that disregard the fact that information is produced, sold, stored, maintained and used, i.e. that it behaves as a commodity. (In particular, this implies that information is a resource, as appears from the fact that - as all resources - it may become scarce.) We claim that treating information like the center of mass of the solar system rather than as a (new) type of commodity has a number of serious negative social consequences, especially where computers are concerned.

Most serious is the implied failure to understand that effective information depends on effective social agreements which bind people together in organized activities. Like everything in society, these only last for a while, and so the information they generate also has a limited life.

Secondly: all goods call crimes into existence (but, it seems, not the center of mass of the solar system). If information - including computer information - is basically like commodities, there will be "information crimes" (as in fact there are). Therefore the popular prejudice about information militates against a truly basic attack on the computer crime problem.

There is also the issue of maintenance. If lumps of information are commodities then they must be maintained if stored (unlike the center of mass of the solar system, it seems). Note that computers make realizing the need for maintenance effort especially difficult: computer information that might be useful to someone sometime is produced in enormous quantities; decay does not make it smell (like decaying vegetables), and it hardly takes visible space.

All this is closely related to the essential modifications that have taken place in the relation of computers with their users: while they were originally designed to perform numerical calculations on behalf of single-person users, computers have gradually been turned into processors of information of any kind on behalf of many-person users. As a result of this shift, computer systems designers have now to face such issues as decision support, authentication and information maintenance.

The word "computer" seems more related to "number" than to "information", and this is historically accurate. But with hindsight we can say: numbers in a computer are always informations, even if this is not the case in mathematics. It is one thing to consider the properties of the number 11 , no matter how it is written (for example the fact that it is a prime); it is another thing to design adequate procedures for dealing with 11 million 
dollars at a bank - or for that matter, 11 pounds of potatoes bought at a market. (In particular, in these latter contexts it does matter how ' 11 ' is written or spoken; because, if a translator is needed, the organized activity is changed.) On the one hand, numbers are (an important) part of the subject of pure mathematics; on the other hand, numbers naturally arise in the context of organized activities - as information, with or without computers. (Numbers also play an important role in applied mathematics as a middle ground between organized activity and mathematics.) Numbers in computers are always information (and never "pure", even if a computer is used by mathematicians for mathematical purposes). Thus we claim: information is the link that ties all computer applications together - including classical computation, a fortiori.

The rest of this paper contains:

- a review of a theory of organized activity which serves as a basis for our definition of information;

- the above mentioned definition of information;

- an indication of practical problems that may be solved with the help of this definition; and finally

- an indication of the relationships between this view of information and other views that are known to us, and some comments on how this view of information impinges on the content of the FRISCO report.

\section{SUMMARY OF A THEORY OF ORGANIZUD ACTIVITY}

What is "organized activity"? According to us, it is a human universal, perhaps even more so than language (for, to communicate by language requires organized activity, but not vice versa). Organized activity can evolve within a society, or it can be artificially created - by design and implementation. Organized activity always presupposes a society, but can be carried out by very few or very many persons. The following "equivalences" will help convey the idea:

2.1 Organized activity is present if and only if coordination (of action) is present.

2.2 Organized activity is present if and only if repetition (of something) is present.

Organized activities, while possibly for fun or for profit or for both, are always motivated - necessarily so since they always require effort, and consequently time. Organized activities characterize all human societies, whether primitive or technological. That information always functions in the 
context of organized activities hardly needs to be stressed in the context of the community of readers of this paper. But our approach to its definition and analytic use depends on a few notions summarized below and exposited more fully in (Holt, 1997).

\subsection{Units, realizations and criteria}

Organized activities depend on agreements between the participants as to what is what and who is who. In the theory of organized activity this is vouchsafed by UNITS which, in their turn - as a social phenomenon - only arise in the context of organized activities and point to an order of reality which stands in between the usual dichotomy subjective/objective and that we call, for lack of a better term, CONJECTIVE. ${ }^{1}$ In many cases units are similar to concepts, being denoted linguistically by common nouns, but much more belongs to the realm of conjective than just concepts. There are units that can only be defined operationally, like e.g."double-click the mouse button", or "clean glassware" in the chemical laboratory, and it would be in harmony with the present view to argue that, in fact, all units are so defined (within a particular organized activity). Units are designed to model the "conjective world" associated with the execution of plans within a community of cooperating performers.

Units of special kinds have received much attention in the social sciences, notably in linguistics and anthropology as for instance phonemes in the sense of structuralist phonology (Trubeckoj, 1939). The whole system of oppositions that defines the phonological structure of a language is produced and maintained by the collective action of the community of speakers. In anthropology, notions like mana and hau, both studied by Mauss (Mauss, 1950), cannot be understood outside those particular organized activities that are the relevant magical rites and ceremonies for the exchange of goods, respectively, and are units in the communities involved. The same dependence of meaning on social practices is highlighted by Malinowski in his analysis of the record of a trading expedition in the Trobriand Islands (Malinowski, 1923). But, of course, it is in Wittgenstein's later philosophy that organized activities (in the form of "language games") are shown to be pervasive and conjectivity is fully recognized as playing a central role even in so formal a practice as arithmetic (Wittgenstein, 1956, 1976).

Each unit is associated with a CRITERION to judge whether some thing or event, real or only imagined, is or is not one of its EXAMPLES. Two units are different if and only if their criteria are different.

We can distinguish between two types of units:

2.1.1 Those for which the occurrence of disagreement represents a plan failure, and those for which it does not. We will call the first type of unit and its criterion DEFINITE; the second type (and its criterion) INDEFINITE.

'This term is formed from 'cum' (together), and the verb 'iacere' (to throw) which provides the common root to 'subjective' and 'objective'. 
Thinking in terms of units (and their criteria) makes it important to be clear about the relationship of units to their examples. Suppose we set up a clerk to sort forms that arrive at his desk into two piles - pink ones on the right, blue ones on the left. In this plan two units play a fundamental role: pink forms and blue forms. Even if this clerk as a matter of fact never sees a blue form, "blue form" is a unit of basic importance to this plan. To handle the resulting distinctions, we introduce the following vocabulary:

2.1.2 A REALIZATION of a unit is an historically real (and therefore irrepeatable) example.

2.1.3 An INSTANCE of a unit is an imagined example; the community may or may not believe that the unit criterion can be met in reality.

The role of set inclusion is played in the present setting by the following intensional notion:

2.1.4 Unit $Y$ is a CASE of unit $X$ if: satisfying the criterion of $Y$ implies satisfying the criterion of $X$, but not vice versa.

In particular, if $\mathrm{Y}$ is a case of $\mathrm{X}$, then every instance of $\mathrm{Y}$ is also an instance of $X$. The following concept will be instrumental later on, in our definition of information.

2.1.5 Unit $X$ has (a set of) CASE ALTERNATIVES if: there is a finite set $Y_{i}$ of two or more cases of $X$ such that every instance of $X$ is an instance of one and only one $Y_{i}$. If unit $X$ has case alternatives, both $X$ and its case alternatives are definite.

\subsection{Bodies and actions}

The theory of organized activity assumes that the reality of organized life can be captured in two types of lumps and their relationships: lumps of effort, called ACTIONS, and lumps of material, called BODIES.

2.2.1 The actions as well as bodies must be conjects for the potential performers, and therefore are instances of units (these units may be called ACTION and BODY, respectively).

The exertion of human effort always "takes time"; similarly every material lump - whether a piece of furniture, a building, or a human body - always "takes space", where "space" and "time" are meant in the sense of ordinary experience. The following notions are motivated precisely by the fact that all units are lumps:

2.2.2 Unit $Y$ is PART of $X$ if: every instance of $X$ includes (as a part) an instance of $Y$. 
2.2.3 Unit $X$ has a DECOMPOSITION if: there is a finite set $Y_{i}$ of two-ormore parts of $X$ such that every instance of $X$ contains one, and only one, instance of each $Y_{i}$; every part of $X$ is included.

These definitions, together with those relative to case alternatives, are helpful in the study hierarchies and classifications. In particular, in many important examples classifications are just a way of establishing mappings between case alternatives and decompositions: think of a computer menu (whose items correspond to case alternatives of an action) or a map of a public transport network, that displays (as its parts) all possible alternative routes.

The effects of an action on a body involved in it is part of the definition of both - the action as well as the body. The effects are various, but two types in particular are especially relevant to the present theory:

2.2.4 PRODUCTION AND CONSUMPTION. An action may CONSUME or PRODUCE a body. These two are mutually exclusive: an action cannot both consume and produce a body. (A fortiori, an instance of an action cannot produce as well as consume an instance of a body.) A body consumed by an action is called an INPUT of the action; a body produced by an action is called an OUTPUT of the action.

Instead of producing or consuming a body, an action can change the state of the body: our definition of a state relies ultimately only upon the notion of case alternatives of a unit.

2.2.5 STATE ChANGE. A STATE $S$ of a body $B$ is a case of $B$ that belongs to a set of case alternatives (2.1.5). A change of state of a body is an action that consumes one case of a body and produces another case of the same body. Thus the body $B$ is present throughout a change of its state; but the action causes $B$ in its input state to vanish, and $B$ in its output state to be born.

\subsection{Plans and exceptions}

A fundamental aspect of the theory of organized activity is this:

2.3.1 Performing an organized activity means: following its PLAN while countenancing EXCEPTIONS.

Thus the theory says: every organized activity is described by a plan, but its actual performance can (and usually does) involve deviations from the plan. ${ }^{2}$ Obviously, practices for the creation, management, change etc. of an

${ }^{2}$ Petri nets may be considered as a graphical language for the expression of plans; so can Diplan (Holt, 1997) and others. 
organized activity is an organized activity itself. Within this latter, PLAN is a unit, whose criterion - of course - must be consistent with 2.3.1. This division of the reality of organized activity into plans and exceptions is of far-reaching importance. It allows us to develop precise analyses based on plans, knowing full well that "real life" is never precise.

Additional units, that relate actions and bodies, play a role in the study of organized activity - namely two types of persons: flesh-and-blood PERSONS and ORGANIZATIONAL ENTITIES - akin to "juridical persons" but more inclusive.

2.3.2 The performance of any action is driven by the INTERESTS of its performers.

2.3.3 Persons are considered to be driven by personal interests; organizational entities by organizational interests.

For a given action, the number of flesh-and-blood persons involved (and their flesh-and-blood identities) may vary from execution to execution of the same plan; it can even vary within a single execution.

\section{INFORMATION}

\subsection{What is it?}

With the help of the above we can now construct a new notion of information. To begin with we assume:

3.1.1 INFORMATION is always carried by a body (the body which carries it being called its CARRIER).

When does a body $B$ carry information $J$ ? The form of this question suggests several matters of importance.

3.1.2 Whether body $B$ carries information $J$, depends on an organized activity within which $B$ has its definition.

Thus, the same physical body may carry information $J$ in one organized activity context, and not in another; similarly, in different contexts it may carry different information.

3.1.3 That information - and even what information - is carried by a body can be part of the criterion of a body. 
And now, the answer to the question:

3.1.4 (a) $J$ is the information carried by a body $B$ if and only if $J$ corresponds to a state of $B$.

(b) The body in this state is operated upon in a manner which one expects of a body carrying information.

To reiterate: it is not enough that $B$ have a state (which might be interpreted as information); the plan must foresee operations performed on $B$ characteristic of information carriers. As far as 3.1.4(a) is concerned, any body might be interpreted as carrying information. So:

3.1.5 INFORMATION (as a unit) is the same as: a body which carries information.

\subsection{Information and meaning}

Claude Shannon (Shannon and Weaver, 1949), helped to develop a technical notion of "information" that is radically divorced from meaning, against the naïve conception. Our own position is quite different from his, but certainly not its opposite.

Information being plan-relative, it simply inherits its relationship to meaning from plans. So: once we understand how plans relate to meaning, we will have understood how information does.

Consider as an example the plan $C$ for a game of catch: I throw, you catch; then you throw, I catch. Although this plan abstracts from many details of particular games of catch (such as the height and sex of the players) still, the criteria of $C$ call for two people throwing a ball back and forth - and this last statement is part of its meaning. Now there are many other types of back-and-forth exchanges that are similar to a game of catch, yet obviously different - for example, a normal type of conversation: I transmit a message to you which you receive (analogous to throw/catch); you in your turn, transmit a message to me which I receive (catch/throw) and so on, into the indefinite past/future. All of these - including the game of catch - can be covered by another plan $A$, of which $C$ is a case. $A$ calls for two people passing an object back-and-forth indefinitely.

So what about $A$ and $C$ 's relationship to meaning? Both plans call for two people (really!); both plans call for a something that is passed back and forth between them, but in $C$ this something must be a ball (really!). But: although in ordinary parlance one would call $A$ "more abstract" than $C$, they are - in our view - both bound to real world meanings in the same way; exactly the way in which - in our view - "information" is bound to meaning. 


\subsection{Information equivalence}

It is enough to ask (and answer): when - relative to a plan - are two body instances equivalent (whether or not they carry information).

3.3.1 The bodies $b_{1}$ and $b_{2}$ are EQUIVALENT within a plan if and only if: for any unit $B$ within the plan, $b_{1}$ is an instance of $B$ if and only if $b_{2}$ is an instance of $B$.

Therefore:

3.3.2 Two instances of INFORMATION are equivalent within a plan if they satisfy 3.3.1.

That is about all that there is to it. Yet, to really understand this, it is necessary to internalize the idea that "information" is treated as relative to an organized activity - perhaps represented by its plan.

For example we may ask: do two ways of writing the same number "give the same information"? If the two ways of writing call for two different modes of reading, then the answer must be "no".

Lewis Carroll, in Through the looking glass, makes this point wonderfully:

"Can you do addition?" the White Queen asked. "What's one and one and one and one and one and one and one and one and one and one?" "I don't know," said Alice. "I lost count."

While in the set-theoretic definition of numbers the White Queen's question would be pointless (because 10 is defined essentially as the unary numeral $1+1+1+1+1+1+1+1+1+1)$, Alice is vindicated by the present account of the use of numerals in plans, as she is by the remarks of Wittgenstein (Wittgenstein, 1956; II 3, 2) on calculations with long numerals and the surveyability of mathematical proofs:

"Now let us imagine the cardinal numbers explained as $1,1+1,(1+1)+1,((1+1)+1)+1$, and so on. You say that the definitions introducing the figures of the decimal system are a mere matter of convenience; the calculation $703000 \times 40000101$ could be done in that wearisome notation too. But is that true?-'Of course it's true! I can surely write down, construct, a calculation in that notation corresponding to the calculation in the decimal notation.'-But how do I know that it corresponds to it? Well, because I have derived it from the other by a given method.-But now if I look at it again half an hour later, may it not have altered? For one cannot command a clear view of it. [...] I want to say: if you have a proof-pattern that cannot be taken in, and by a change in notation you turn it into one that can, then you are producing a proof, where there was none before."

Thus, the difference between notations is seen here, quite appropriately, not as a subjective matter related to the psychology of perception, but as a conjective fact on which is based the execution of a plan. 


\subsection{Commodities and information}

Commodities are relevant to information because information is often packaged as a commodity, and therefore is subject to commodity-relative operations - such as trade, transfer from owner to owner, storage and (ultimately) use. Computers are used extensively to support such commodity-relative operations as applied to packages of information. It follows that computer implementation of information handling must also address commodity-relative operations, as we shall now try to illustrate concretely.

Take as an example the problem of AUTHENTICATION - a problem that: (a) is very real, especially in the context of computers; (b) makes no sense if information is deprived of its market setting; (c) is also a problem for non-informational commodities.

Why does the authentication problem exist? Because, under most modern circumstances: (a) the commodity passes through many intermediate hands so variable with time and user location, that the user cannot keep track of the intermediaries; (b) there are many possible producers, ingredients, and methods for any given commodity as it presents itself to the user. Thus:

3.4.1 The package of the commodity may carry features which identify the producer and/or the ingredients and/or the methods of production. These latter we shall call IDENTITY FEATURES of the commodity.

3.4.2 User and producer - who after all depend on each other - are interested in binding the identity features to the contents so that: (a) the binding can be performed without a lot of expense and trouble; (b) a potential criminal would find tampering with this binding too expensive to be worth the trouble.

3.4.3 The identity features should make it easy for the user to contact the producer, or to judge if the ingredients and methods suit his purposes.

Computers were never designed to facilitate the binding of identity features to information packages (3.4.2); nor were they designed to ease the use of identity features in allowing the user (of information) to contact its producer (3.4.3).

\subsection{Operations characteristic of information}

We have seen in 3.1.4(b) that a defining feature of information is the set of operations that can be performed on a body that carries information. These are of the following three kinds:

3.5.1 MATHEMATICAL TRANSFORMATIONS, of the sort used in logic, copying and coding. 
3.5.2 DECISIONS, like those needed to answer questions like "is the variable $x$ greater than 0 ?" or "To what place should this letter be sent?"

3.5.3 CONVERSIONS, like those performed in operating numerically controlled machines, or in producing visual images from computer programs.

While mathematical transformations produce information out of information (except for copying from a medium to another one, in which case one should rather speak of a conversion), decisions take information as input and produce actions. On the other hand, conversions normally rely on peripherals (for typing and display) and can be regarded as special kinds of decisions, because they rely essentially upon them. We shall not dwell in detail upon these operations, but illustrate here the kind of problems that arise in connection with two especially important examples, namely copying and deciding.

While it is true that many physical objects (such as houses) can be copied, copying is particularly effective (mechanizable, and therefore made fast and cheap) in the case of information. In all copy operations - whether of information or of houses - there is an "original" which is not destroyed as input, and one or more "copies" which are produced as output, of the copy operation. The framework for a theory of organized activity set up above naturally raises the question: does one copy a unit or a realization of a unit? We assert: only units can be copied (or transmitted), and not realizations.

As an illustration of how the argument runs, consider copying a document page by means of a fax. Since bits are units, so is a bit stream. There is no doubt, therefore, that a new instance of the stream of bits created in a fax sender can be recreated in a fax receiver. But a stream of bits - no matter how many they are - can at best specify a unit of which the document page is a realization. Therefore, the page to be transmitted will certainly have properties that the stream of bits will not characterize. Assume that we produce the page with a machine that exercises dimensional control over the characters beyond the capabilities of normal fax printers. Suppose, further, that use is made of this dimensional control to encode a secret message that does not depend on the character content of the page. The meaningful "information" on such a page obviously cannot be transmitted by fax. Now, it is enough to get a fax with finer resolution, so we can put the message on a page over which it is possible to exercise humidity control; the degree of humidity is not encoded. Could it be theoretically? Yes. But we claim that it is not theoretically possible to list in advance all properties that might matter, so that these too can be encoded.

And now deciding: it is a fundamentally important reason for the intimate tie between information and organized activity; it is also an important part of the reason why computers are so crucially important to our society.

We will call an action a DECISION if one of its consequences is choosing one of several possible next actions - whatever other 
consequences there may be. A decision always resolves a choice between several next action alternatives. Thus a restaurant customer, on reading the menu (input information) does not - technically speaking - choose between steak and lobster, but chooses to order steak, or to order lobster. As in the case of all actions, decisions take effort (on the part of some actor), and they require one or more packages of information as input. Decision is an end use of information; a decision may or may not consume its informational inputs. Whether something is, or is not, a package of information that enters a decision may depend on relatively sophisticated considerations - as illustrated by the following contrastive examples.

At some point in time, a door is locked, or left open. After this, actor $X$ comes by.

a) $X$ may push on the door: if the door is not locked, $X$ goes through; if the door is locked, $X$ is prevented from advancing;

b) $X$ may try the door to decide whether it is, or is not, locked; choose his next action accordingly.

In case a) the state of the door is not informational (relative to $X$ ); in case b), it is.

As a (somewhat) elaborate illustration, suppose $A$ in city $X$ sends a letter to $B$ in city $Y$. $A$ puts the letter in a mailbox. The letter is an information package. At the first step - that of transporting the letter from the mailbox to the post office, all of the information that the letter carries exercises no influence. At the next step a rough-sort of letters takes place according to general destination. At this stage a part of the address on the envelope contributes to a decision; the rest of the address (name and street address of recipient) and the content continue to have no influence. The mail delivery person decides on how to handle this letter based on the rest of the address which, at this stage, is input to his decision; the recipient who opens the letter and reads it, uses the informational content of the letter in some combination of ways which is hard to specify. The sender's address and the cancellation stamp affixed by the post office may serve to control the letter source, and the time of its mailing. If this becomes necessary, they too will be input to decisions made at a later time.

At the dawn of modern science, a French schoolman, Jean Buridan, took a serious look at human decision, in the form of the following question:

If a hungry man be placed at equal distance from two equally attractive dishes, will he starve to death?

Contrary to common sense, Buridan thought the right answer was "yes" (he would starve to death). As we read his answer, it should be taken to mean:

- The choice of dish should depend on the two dishes, and on nothing else.

- In all relevant respects, the two dishes are assumed the same. 
- Therefore, given that the hungry man only looks at the two dishes, the combination offers him no information on the basis of which to make a choice (make a decision) - and a decision cannot be made without information, the very same assumption that we make.

We want to emphasize that decisions taken in the context of a computer program are not the same as decisions taken by persons. For example: a person may, in the eyes of others, make a wrong decision; others will decide that his error was due to inattention, willful sabotage, etc., and he will be reprimanded, or otherwise punished, accordingly; a computer program is not judged in the same way, nor will the computer program be reprimanded or punished. Nevertheless, everything we have said above applies with equal force to decisions carried out by persons pursuant to their roles, or decisions carried out in programs on behalf, and under the responsibility, of some actor.

\section{RELATED WORK AND CONCLUSIONS}

The organizational theory of information sketched here has several points in common with the work described in the FRISCO report (Falkenberg et al., 1998): in particular, it aims at the same thing by related means; yet there are fundamental differences that are worth stressing. Both approaches emphasize the need for a general philosophical outlook, but a first divergence appears already in the choice of the subject matter: while information systems are the defining concern of the FRISCO project, we believe that 'system' is not necessary to the analysis of information. Rather, the place of systems in our approach is held by organized activities that, as we have suggested, are the environment in which most naturally information is produced and consumed. Also the theory of information described in the FRISCO report points, though implicitly, to a theory of organized activity - via the definition of such terms as organisational system, actor (as a responsible thing), goal. In fact, the conceptual framework of the theory of organized activity overlaps to a large extent with that of the FRISCO report, though the underlying ontological assumptions seem to differ substantially. For example, "social reality" is construed there as the result of communicating (representations of) conceptions that are based on individual cognitive activity (Falkenberg et alii, 1998; Assumption [h]). For us, organized activity underlies the formation of units, which in their turn, create the conjective world. (Observe that even the term 'inter-subjectivity', used in FRISCO with a meaning often similar to that of 'conjectivity', implies a prior understanding of 'subjectivity', while conjectivity claims to be a primary fact - more basic than either objectivity or subjectivity.) 
Because of this more operational attitude, our views are perhaps closer to the position of Stamper, sketched in (Falkenberg et alii, 1998) and in related publications (Stamper, 1985, 1996). He emphasizes the social aspects of information systems, and its ontology, uses Gibson's notion of affordance, an invariant repertoire of behavior, that seems to have a conjective flavor. Social affordances, those that make up social reality, arise then as the invariants of organized behavior. Also, his proposal to root the study of information in the notion of sign seems to make signs similar to the units of the theory of organized activity sketched above.

Another far-reaching alternative to traditional theories of information is that described in (Goguen, 1996), where the prominence given to the social (and ethical) aspects of information is accompanied by the recourse to concepts and techniques taken from the social sciences, in particular ethnomethodology. There, the slant on conjectivity shows up in the need for a social semiotics, as opposed to the traditional logical semiotics that lies behind either the statistical theory of information (Shannon and Weaver, 1949) or descriptive theories based on model-theoretic semantics, like (Barwise, Perry, 1983) and (Barwise, Seligman, 1998). (This is also a main concern in (Stamper, 1996), appearing in the upper levels of his "Semiological Ladder" and motivating the fundamental role played by the concept of a norm in his account of information systems.)

A leading unifying theme in all these views is a natural consequence of the fact that information is always situated in a group involved in an organized activity of some kind: namely that responsibility enters the processing of information. The members of such a group are responsible for maintaining the criteria for judging whether something is an example of some unit (Holt, 1997); signs have to be interpreted by someone, who becomes responsible for that interpretation (Stamper, 1996); the members of the group are held accountable for the interpretation of signs as describing an item of information, as for the Principle of Accountability in (Goguen, 1996), derived from ethnomethodology. ${ }^{3}$

This paper has focused mainly on those aspects of the theory of organized activity and of information that may be useful to information system designers, rather than users. The concepts that the present theory helps to formulate can, however, also be applied by system users in many cases of practical interest. In particular, there is a theory of hierarchies (and classifications) based on the first principles of the theory of organized activity, that relates bodies with interests, and provides a new view of the organization of computer storage. There are other delicate topics in the study of information that we haven't directly addressed in this paper. Notably, all

${ }^{3}$ A closer investigation of this discipline is needed in order to see whether the notions of category and method used there are coextensive with those of unit and criterion. 
uses of information in documents (especially contracts) are of great importance for computer applications used in banking and electronic commerce. In part these uses are accounted for by viewing information as a resource, and by putting it in the framework of an economic process, but a smoother treatment of these problems needs a detailed study that will be pursued elsewhere.

\section{REFERENCES}

Barwise, J., Perry, J.: Situations and Attitudes, MIT Press, Cambridge (Mass.), 1983.

Barwise, J., Seligman, J.: Information Flow, Cambridge University Press, Cambridge (UK), 1998.

Diamond, C.: Wittgenstein's Lectures on the Foundations of Mathematics, Cambridge 1939, The University of Chicago Press, Chicago and London, 1976.

Falkenberg et alii: A Framework of Information System Concepts, 1998. Web edition available at: ftp.leidenuniv.nl/pub/rul/fri-full.zip.

Goguen, J. A.: Towards a social, ethical theory of information, in Social Science Research, Technical Systems and Cooperative Work: Beyond the Great Divide, edited by G. Bowker, L. Gasser, L. Star and W. Turner, Erlbaum, 1997, pp. 27-56.

Holt, A. W.: Organized Activity and Its Support by Computer, Kluwer Academic Publishers, Dordrecht, Boston and London, 1997.

Kripke, S. A.: Wittgenstein on Rules and Private Language, Basil Blackwell, Oxford, 1982.

Malinowski, B.: The problem of meaning in primitive languages, Supplement I to: Ogden, C.K. and Richards, I. A., The Meaning of Meaning, Harcourt, Brace and Jovanovich, San Diego, New York and London, 1923.

Mauss, M.: Sociologie et anthropologie, Presses Universitaires de France, 1950. (A collection of essays, including Esquisse d'une théorie générale de la magie, 1902-3, and Essai sur le don: Forme et raison de l'échange dans les sociétés archaïques, 1923-4.)

Shannon, C., Weaver, W.: The Mathematical Theory of Communications, University of Illinois Press, 1949.

Stamper, R. K.: A logic of social norms, for the semantics of business information, in Knowledge and Data, edited by T. Steel and R. Meersmann, North-Holland, 1985.

Stamper, R. K.: Signs, Information, Norms and Systems, in Signs at Work, edited by B. Holmqvist, P. B. Andersen, H. Klein and R. Posner, De Gruyter, 1996.

Trubeckoj, N. S.: Grundzüge der Phonologie, Travaux du Cercle Linguistique de Prague, VII, 1939; Vandenhoeck \& Ruprecht, Göttingen, $1958^{2}$.

Wittgenstein, L.: Bemerkungen über die Grundlagen der Mathematik, edited by G. H. von Wright, R. Rhees and G. E. M. Anscombe, Basil Blackwell, Oxford, 1956. English translation by G. E. M. Anscombe. 